\title{
Über die Geburt der Antike aus dem Geist der Moderne
}

\author{
DIETRICH HARTH
}

To recall the shaping of "antiquity" as a leading symbolic concept of European culture seems to be justified by the actual debate about the fault lines between modernity and postmodernity and emerging cultural fundamentalisms. In 17th century France the "Querelle des Anciens et des Modernes" fostered not only the consciousness of historical distance between now and then but brought forth the image of an enhanced antiquity, a "new Rome". The resulting hegemony of the "esprit classique" in central Europe provoked the opposition of intellectuals in 18th century Germany searching for national cultural identity. In the dramatic course of this search for self-constitution Greek antiquity was elaborated as a symbolic token ("reine Griechheit") for a cultural pattern that was deemed to overthrow and even transcend universally the impact of French culture. The construction of antiquity as a cognitive image (Denkbild) with aesthetic features can be considered as a response to the question if the antagonistic structure of modern life could be overcome by a utopian idea restricted, however, to a relativistic, i.e. national concept of classical studies.

\section{Aktuelle Dimensionen des Themas}

prechen wir heute von "der Antike", so setzen wir-ohne daß uns das immer $\checkmark$ bewußt ist-voraus, daß dieser Name der Welt, die er benennt, unbekannt war. Denn dieser Name, der doch etwas sehr Fernes bezeichnet, hat ein geradezu intimes Verhältnis mit "der Moderne" ${ }^{1}$ Das heißt aber: mit jener neuen Welt, die glaubt, im reifen, erfahrungsgestählten Mannesalter auf die Antike als Kindheit und Jugend zurückblicken zu können. ${ }^{2}$ Im Denken und Dichten der durch historische Kämpfe und Differenzierungsprozesse belehrten Bewohner der Neuzeit erscheint die perspektivisch zur Ruhe gebrachte und in eine symbolische Ordnung aufgehobene geistige Welt der

Nach einem Vortrag gehalten auf der 2. Tagung der International Society for the Classical Tradition, Tübingen, 13.-16. August 1992.

1. Zu Entstehung und Bedeutungswandel der Leitbegriffe vgl. die Artikel "Antike" in: Historisches Wörterbuch der Philosophie, ed. J.Ritter, Bd.1, Darmstadt 1971, 385-392, und "Modern, Modernität, Moderne", in: Geschichtliche Grundbegriffe, ed. O.Brunner et al., Bd.4, Stuttgart 1978, 93-131. S auch Jacques Le Goff: Geschichte und Gedächtnis, Frankfurt/New York 1992, $49-82$.

2. Für viele Autoren des 18.Jh.-etwa für Thomas Blackwell und Joh. Gottfried Herder-war die historiographische Kindheitsallegorie positiv besetzt; sie diente dazu, die erwünschte Ähnlichkeit zwischen Altem (Kind) und Neuem (Mann) zu begründen, und ist symptoma-

Dietrich Harth, Germanistisches Seminar, Universität Heidelberg, 69117 Heidelberg Germany.

International Journal of the Classical Tradition, Vol. 1, No. 1, Summer 1994, pp. 89-106. 
Griechen und Römer wie ein unerschöpflicher Fundus der Parolen und Masken, die sich lange genug im je aktuellen Ideenstreit als nützlich erwiesen. In "der Antike" - so lautete ein geläufiger Spruch-soll die Geburtsstunde des europäischen Geistes-der Künste und Wissenschaften-geschlagen haben. Und wer diesen Geist beschwört, steht schon in der Schuld der Alten, hat schon über die fundamentale Differenz zwischen Damals und Jetzt hinweg den Bogen der Analogie, wenn nicht der Genealogie geschlagen. Woher kommt das? Was sind die Gründe?

Die ersten Spuren für die auf Tiefenschärfe bedachte Unterscheidung zwischen "dort die Antike-hier die Moderne" reichen zurück in den Frühhumanismus. ${ }^{3}$ Schon in dieser historischen Situation läßt sich das intellektuelle Muster in Umrissen erkennen, das die (Wieder-)Aneignung/Erfindung der Antike unter den Bedingungen eines gebrochenen Zeitbewußtseins durchzieht: Negation der geltenden Ordnung unter Rückgriff auf eine zwar heterodoxe, aber nicht gänzlich fremde kulturelle Semantik. Erst im Barock jedoch löst die europäische Geschichtsschreibung die heilsgeschichtliche Prozession der biblischen Monarchien vollends auf: Altertum und moderne Zeit werden nun als exponierte, mit klaren Kennzeichen versehene Altersstufen unterschieden, zwischen ihnen ein vergleichsweise diffuses Mittleres. Und trotz des unübersehbaren Abstandes soll die moderne der antiken Welt näher als der des Mittelalters stehen. Oft genug, doch letztenendes erfolglos ist diese Dreiteilung zusammen mit ihren relativistischen Wertsetzungen kritisiert worden. Sie scheint bequem genug, um ein Bedürfnis nach triadischen Denkfiguren zu befriedigen und zugleich damit die Überlieferung wie ein dreigeschossiges, mit einem Blick zu umfassendes Gebäude überschaubar, sie erinnerungs- oder vergessensfähig zu machen.

Die beständige Aneignung und unaufhörliche Neu-Interpretation der antiken Kultur gehört noch in unserer Gegenwart zu den widerstandsfähigsten Bestandteilen der staatlich dotierten Gedächtnispflege in Akademien, Universitäten, Museen. Diese Arbeit dient-so scheint es-dazu, den harten Kern der europäischen Kulturüberlieferung zu wahren, an dem sich die Kritiker jenes Segregationsbewußtseins die Zähne ausbeißen, für das die Differenz zwischen europäischer "Kultur" als exklusivem Einheitsbegriff und außereuropäischen fremden, also exkludierten "Kulturen" selbstverständlich ist. Es ist nicht zu verkennen: Die in der Modernisierungsperspektive als identifikationsfähige und universelle symbolische Ordnung konstruierte "Antike" hat die Ab- bzw. Ausgrenzung von nicht dazugehörigen, z.B. orientalischen, asiatischen, afrikanischen Kulturen überhaupt erst möglich gemacht. Sie ist andererseits ein Ferment jener Modernisierung, die den Prozeß der die Sache erinnernden und zugleich transzendierenden Aneignung der Antike reflektiert, der zu Beginn der Neuzeit in Gang kommt, um im frühen 19. Jahrhundert in die arbeitsteiligen, professionalisierten Strukturen der Kulturwissenschaften eingebettet zu werden.

In dem folgenden Essay möchte ich einige Materialien aus dem Text-Archiv

tisch für die genetische Strukturformel des europäischen Philhellenismus. Vgl. auch Norbert Miller: "Europäischer Philhellenismus zwischen Winckelmann und Byron". In: Propyläen Geschichte der Literatur, 4.Bd.: Aufklärung und Romantik 1700-1830, Berlin 1983, 315ff. Noch Marx, der sein Antike-Verständnis zugleich an Winckelmann und den Junghegelianern geschult hat, macht vom Kindheitsbild Gebrauch, um die ihm merkwürdige, anscheinend a-historische Geltung des klassischen Ideals zu umschreiben. Dazu Panajotis Kondylis: Marx und die griechische Antike, Heidelberg 1987, 64ff.

3. Eckhard Kessler: Petrarca und die Geschichte. Geschichtsschreibung, Rhetorik, Philosophie im Übergang vom Mittelalter zur Neuzeit, München 1978. 
dieser Konstruktions- und Erinnerungsarbeit zusammentragen. Es sind dies Materialien, die sich auf wenige dominante Strukturen und Prozesse in der Geschichte vor allem Frankreichs und des deutschen Sprachraums im 17. und 18. Jahrhundert, im Zeitalter der Aufklärung und des Idealismus, beziehen. Dieses an Verallgemeinerungen interessierte Vorhaben ist umständlich, und deshalb komme ich um Vereinfachungen nicht herum. Vereinfachungen vor allem begrifflicher Art, die keinerlei Originalität beanspruchen. Mein Vorgehen verstehe ich eher wie das des Schuldeneintreibers, der einst in der englischen Tradition die Berufsbezeichnung "remembrencer" trug."

Beginnen wir mit einem kurzen Blick auf die heute verbreitete Semantik des Modernisierungsbegriffs, der den mit westlichem Ideengut verknüpften Begriff der "Aufklärung" in auffallender Weise ersetzt hat. Es waren die Sozialwissenschaftler (vor allem in den USA), die nach dem Zweiten Weltkrieg und nach der gleichzeitigen Entkolonialisierung den allgemeinen historischen Wandel von Gesellschafts- und Wirtschaftsstrukturen unter dem Begriff der Modernisierung zum Thema gemacht haben..$^{5}$ "Modernization" wurde von ihnen nicht selten als "Westernization" gedeutet: Es ging zunächst und allererst um die Evolution und den künftigen Status der eigenen Gesellschaften innerhalb der miteinander konkurrierenden entwickelten Systeme und dann erst-in einem zweiten Schritt-um die Frage, ob und wie die außerwestlichen, die sog. nicht-entwickelten Gesellschaften am westlichen Entwicklungsmodell zu orientieren sind. Modernisierung wurde hier also als Universalie verstanden, die überall in der Welt Geltung besitzt und mit positiv aufgeladenen Wertideen gleichzusetzen ist: z.B. Überwindung gesellschaftlicher Ungleichheit durch kodifizierte Gleichheitsgarantien, Demokratisierung der traditionellen politischen Herrschaftsstrukturen, Partizipation aller Gesellschaftsmitglieder an den Entscheidungen der Politik, ökonomisches Wachstum, kulturelle Alphabetisierung bis in die Peripherie sozialer Felder und anderes mehr. Der Begriff der Modernisierung hat in dem skizzierten Rahmen neben einer deskriptiven unverkennbar auch eine normative Bedeutung: Er bezeichnet die stets zu vollbringende Einlösung jener praktischen Ideen einer am Gleichheitsprinzip orientierten gesellschaftlichen Evolution, die im Zeitalter der europäischen Aufklärung ihre Ursprünge hat und in den Menschenrechtserklärungen mit postkonventionellen Geltungsansprüchen kodifiziert worden sind.

Inzwischen aber hat diesen ja noch sehr jungen, diesen optimistischen Modernisierungsbegriff eine eher skeptische Vorstellung abgelöst. Eine ironische Umkehrung im Verhältnis von Moderne und Tradition ist an die Stelle des früheren Optimismus getreten. Diese läßt sich am eindrucksvollsten mit dem Einwand umschreiben, die optimistische Geschichte der Modernisierung produziere nichts anderes als einen neuen Mythos, der die Tatsache verschleiere, daß eben das, was diese optimistische Geschichte feiert, zur Vernichtung alternativer außerwestlicher Kulturen und-was genauso wichtig-zur Stärkung eines anonymen, nichtsdestoweniger praktisch äußerst wirksamen staatlichen Gewaltmonopols und damit zugleich zur Schwächung individueller Verantwortung beigetragen habe. Dieser skeptische Standpunkt wirft einen tiefdunklen Schatten auf die optimistische Idee der durch Modernisierung einzulösenden Veränderung des Alten und scheint nach Alternativen in der Bewahrung heterodoxer und

4. Hinweise und Anregungen verdanke ich den Arbeiten von Hans Robert Jauss, insbes. dem Aufsatz "Literarische Tradition und gegenwärtiges Bewußtsein der Modernität", in: Ders., Literaturgeschichte als Provokation, Frankfurt/M. ${ }^{31973,11-66 . ~}$

5. Vgl. etwa die von S.Rokkan herausgegebenen Beiträge in: Comparative Research across Cultures and Nations, Paris 1968. 
fremdkultureller Traditionsbestände und nicht in deren permanent fortschreitender Überwindung zu verlangen. Das Rationale schlägt um ins "Irrationale", in einen neuen Mythos: So sprechen die entscheidungsindifferenten Beobachter dieser Entwicklung vom Eintritt in die postmoderne Sinnleere und antworten darauf mit einer ästhetischen Therapie, die im unverbindlichen Zitat ornamental erinnert, was der Modernisierungsprozeß funktionalisiert oder hinweggeschwemmt hat. ${ }^{6}$ Die unbeirrbaren Wahrheitssucher klammern sich in dieser Situation an eine Denkfigur, die ihrerseits zu den Elementen des europäischen Modernisierungsprozesses gehört: Sie rufen nach den Rehabilitierung vormoderner, insbesondere antiker, z.B. neoaristotelischer Problemlösungen auf der Ebene ethischer, über die Zukunft des Planeten entscheidender Fragen. ${ }^{7}$ Dem steht entgegen, daß sich "die" Menschheit längst nicht mehr entlang den Grenzen westlich-abendländischen Denkens definieren läßt. Modernisierung ist auch ohne Verwestlichung, ja soger gegen das westliche Weltbild möglich. Der Begriff löst sich mithin aus der-sei es komplementären, sei es antithetischen-Koppelung an das Gegenbild des Alten, des Traditionellen bzw. der Antike, um mit ganz verschiedenen Kulturmustern sich zu verbinden. Es ist nicht auszuschließen, daß diese globale Diversifikation des Modernisierungsprozesses zu Zivilisationskonflikten führen wird, in deren Verlauf die Legitimationsstragien der Konfliktparteien wieder auf die ältesten Gehalte ihres Kulturkanons zurückgreifen werden. ${ }^{8}$

Natürlich setzen alle optimistischen oder auch bloß deskriptiv gemeinten Modernisierungsgeschichten etwas voraus, das sich von der Moderne-hier als makrostruktureller Epochenbegriff im Sinne eines offenen Systems gebraucht-augenfällig unterscheidet; genauer: etwas, das bis in die Einzelheiten des habituellen Lebensstils als eine Gegenposition erscheinen muß. Dieses im Verhältnis zur Moderne ganz andere heißt je nach historischem Standort: "Antike" oder neutraler: "Tradition". Es ist trivial, aber nichtsdestoweniger zutreffend: Was solche Begriffe- " Antike" und "Tradition" bedeuten, das hängt von denen ab, die sich als "Moderne" von Ursprungsdenken und Traditionalismus unterscheiden wollen. Und so ist denn im Grunde meine Frage nach dem Begriff der Modernisierung eigentlich schon beantwortet.

Ein so dürftiges Ergebnis befriedigt niemanden. Die Frage bedarf, um übers Triviale hinauszukommen, der historischen Konkretisierung, will sagen: einer eingehenderen Betrachtung der Ideenkämpfe, die auf der Grenzlinie zwischen Tradition und Moderne geführt worden sind. Ich möchte im folgenden an einigen historischen Beispielen zeigen, welche Bilder der Antike und welche Konstruktionsmechanismen im Untersuchungszeitraum zwischen etwa 1650 und 1810 aus diesen Kämpfen hervorgegangen sind, wie sie schließlich das Alte in jene Alterität transformierten, an der die Epoche der Moderne einerseits ihren eigenen, dauernd fortschreitenden und permanent sich verändernden offenen Prozeßcharakter, andererseits aber so etwas wie die Orientierungspunkte in einem Fluß permanenten Wandels ablesen konnte. Meine Belege beziehe ich hin und wieder ohne strenge Quellenanzeige aus z.T. weit auseinanderliegenden wissenschaftlichen Archiven, und ich folge streckenweise den Schlüs-

6. Siehe die kritischen Analysen von Zygmunt Bauman: Intimations of Postmodernity, London 1992.

7. Franco Volpi: "Praktische Klugheit im Nihilismus der Technik: Hermeneutik, Praktische Philosophie, Neoaristotelismus", in: Internationale Zeitschrift für Philosophie 1 (1992), 5-23.

8. Daß sich daraus eine neue Qualität globaler Zivilisationskonflikte ergeben könnte, vermutet Samuel P.Huntington: "The Clash of Civilizations?", in: Foreign Affairs 72/3 (1993), 22-49. 
selbegriffen einer teils systemisch, teils historisch interpretierenden kultursoziologischen Diskussion. ${ }^{9}$ Drei Merkmalsbereiche des Modernisierungsbegriffs lassen sich aus dieser Diskussion herausschälen. Danach bedeutet Modernisierung:

1. Genese eines neuen Zeitbewußtseins, das den dynamischen Wandel als seine eigene Bedingung entdeckt. Stichwort: Historisierung von Vergangenheit und Gegenwart.

2. Genese einer neuen Einstellung zur Kultur als der mundanen Selbstschöpfung der menschlichen Gattung. Stichwort: kulturelle Universalisierung.

3. Genese einer neuen Form der Erfahrung, die-als ästhetische-Natur und Artefakt unter den Bedingungen ihrer Erscheinungsweise, also als ein Reflexionsprodukt und nicht als kultisches Zeichen, als Illustration, als schönes Dekor oder bloße Einkleidung wahrnimmt. Stichwort: Ästhetisierung.

\section{Antike und Moderne im Wechselspiel}

In der Geschichtsschreibung seit dem Altertum war es üblich, die Zeiträume von den ersten Anfängen der Zivilisation bis in die jeweilige Gegenwart nach Lebensalterstufen zu ordnen: von der Kindheit über Jugend und Mannesreife bis zum Greisenalter. Erst relativ spät in der europäischen Kulturgeschichte-im Frühhumanismus des 14. Jahrhunderts-kommt (wie schon gesagt) eine neue Form der Periodisierung auf, die am Ende des 17. Jahrhunderts zwischen Antiquitas - der später so genannten "Antike" - dem Mittleren Alter (medium aevum) und einer Neuen Zeit unterscheidet. ${ }^{10}$ Was in einer langen Generationenfolge verwandtschaftlich verbunden blieb, wird-wie der agrarische Sippenverband durch die Quartiere der Stadtgesellschaft-in die Topographie selbständiger Geschichtsprovinzen aufgelöst: ein erster Schritt zum Relativismus.

Die Denker der Neuen Zeit nennen sich "moderni" nach der lateinischen Vokabel "modernus", die nichts anderes als "jetzt" oder "neu" bedeutet. Sie setzen sich damit von den "antiqui" ab, von den "Früheren" oder "Älteren" (lat. ante). Das klingt plausibel. Denn die Scheidung von Alt und Neu, von Damals und Jetzt, von Reife und Jugend gehört-so möchte man meinen—zum Grundbestand einer individuellen Lebensgeschichte und ist-bei vergröberter Optik-auf die kollektive Kulturgeschichte übertragbar.

Die Unterscheidung von "modern" und "antik" beruht auf einer einfachen Korrelation und erfüllt eine andere Funktion als die historiographische Zeitalterrechnung. Sie steht für ein Drama von Identitätssuche und Sich-unterscheiden-Wollen, das mit der Suche nach einer symbolischen Ordnung zu tun hat, in deren Zentrum der autonom handelnde Mensch steht. Es ist für gewöhnlich die Aufgabe der intellektuellen Eliten in einer Kultur, diese Autonomie gegen die Unvollkommenheiten der hier und jetzt existierenden Ordnung einzuklagen. ${ }^{11}$

9. Shmuel N.Eisenstadt: Tradition, Change, and Modernity, New York/Sydney/Toronto 1973. Richard Münch: Die Kultur der Moderne, 2 Bde., Frankfurt/M. 1986.

10. Christoph Cellarius: Historia Universalis [ . . . ] in antiquam et medii aevi ac novam divisa, 16851696. Vgl. dazu Ulrich Muhlack: Geschichtswissenschaft im Humanismus und in der Aufklärung. Die Vorgeschichte des Historismus, München 1991, $122 \mathrm{f}$.

11. Vgl. dazu die systematische, auf den Wandel der Hochkulturen bezogene Skizze von S.N. 
In der Spätphase der Renaissance entsteht eine Vergleichsliteratur, die im Laufe des 17. Jahrhunderts vor allem in Frankreich, in der "Querelle des Anciens et des Modernes" - im sog. "Streit um den kulturellen und wissenschaftlichen Vorrang der Neueren gegenüber den Alten" —öffentliche Resonanz findet. Verglichen werden in dieser Diskussion die intellektuellen und künstlerischen Leistungen der alten, d.h. der griechischrömischen, und der neuen, d.h. der gegenwärtigen Welt. Parteien bilden sich heraus: dort die Antiqui als Vertreter der alten, vorbildlichen, hier die Moderni als Vertreter der fortschreitenden, vom Alten sich lösenden Wissensinhalte, Wertideen und Verfahrensweisen. Mit einem Wort: die Jetztzeit entdeckt sich selbst als etwas, das anders ist als das vorhergehende, das zeitlich Frühere, das autoritätsbesetzte Wissen, das der Gegenwart so lange als nachahmenswert galt. "Jetzt" im Verhältnis zu "Früher", das ist ein Thema, das nicht nur auf einen Bruch in der Zeit, sondern vor allem auf das Vermögen hinweist, via negationis kulturelle Differenzen zu statuieren, aus deren Konflikten eine neue, vollkommenere Ordnung entstehen soll. Im Verlauf der "Querelle" werden Antworten auf die Frage nach den Orientierungsmaßstäben für die neu entdeckte, die Jetztzeit gesucht: ob die Gegenwart diese aus sich selbst entwickelt-Standpunkt der radikalen "Moderni" —oder ob sie diese mit neuer Begründung der Tradition entnimmt-Standpunkt der "Antiqui". Ob zukunfts- oder vergangenheitsorientiert-der Kampf der intellektuellen Parteien führt zur besseren und genaueren Bestimmung differierender symbolischer Ordnungen, deren Verhältnis untereinander durch Abgrenzung neu festzulegen ist.

Der Vergleich zwischen Antike und Moderne war zwar ein gesamteuropäisches Thema. Doch rief es vor allem in jenen Gesellschaften die heftigsten und folgenreichsten Kontroversen hervor, deren politische und kulturelle Eliten besonders aufwendige Projekte betrieben. Und das waren Projekte, deren gleichzeitig oder rückblickend zugeschriebene Epochentitel offenkundig den Wettbewerb mit der Antike, zugleich aber auch die Ablösung von einem überkommenen Weltbild suchten: Spaniens "Siglo de Oro"-eine Anspielung auf den antiken Mythos vom Goldenen Zeitalter-, Frankreichs "Siècle classique", Englands nach dem römischen Kaiser Augustus so genanntes "Augustan Age". In der Folge dieser Projekte, die etwa in Frankreich unter dem Absolutismus Ludwigs XIV. einen Modernisierungsschub einleiteten, indem sie zur Rationalisierung und Universalisierung der Künste, der Wissenschaften, der Politik, der Ökonomie und des höfischen Lebensstils beitrugen, wurde die Jetztzeit als Höhepunkt gegenüber der unmittelbaren Vergangenheit aufgewertet und in ein produktives Konkurrenzverhältnis zur römischen Antike gesetzt; zu einer kulturellen und politischen Ordnung, aus deren vielfältigen Leistungen die Epoche der Jetztzeit ein mächtiges kulturelles Normensystem herausbuchstabierte. ${ }^{12}$ "Antique" hießen dementsprechend diejenigen Dinge, die-so schrieb der Gelehrte Thomas Corneille in seinem Dictionnaire von 1694-von der modernen Zeit nicht mehr gebraucht werden, ${ }^{13}$ ein Hinweis auf eine

Eisenstadt: "Die Mitwirkung der Intellektuellen an der Konstruktion lebensweltlicher und transzendenter Ordnungen", in: A.Assmann/D.Harth (Hg.): Kultur als Lebenswelt und Monument, Frankfurt/M. 1991, 123-132.

12. Zur ethnozentrischen, also relativistischen Bedeutung des mit dem französischen "esprit classique" verbundenen Universalismus vgl. Tzvetan Todorov: Nous et les autres. La réflexion française sur la diversité humaine, Paris 1989, 19ff. Zur Geschichte des "esprit classique" nach wie vor unentbehrlich die Untersuchung von René Bray: La formation de la doctrine classique, Paris 1926.

13. Thomas Corneille: Le dictionnnaire des arts et des sciences, Tome I, Paris 1694. Eine ähnliche 
nicht nur zeitliche Distanz, da der vom praktischen Bedürfnis befreite Gegenstand sich umso eher für historisierende Betrachtungen eignet.

Die Konkurrenz mit dem, was nicht mehr in Gebrauch-also "antik" -war, ist "produktiv" nicht nur im Sinne der Selbstbestimmung der Gegenwart zu nennen, sondern auch im Hinblick auf die Konstruktion eines historischen Kreislaufs, in dessen Bewegungsbild die Phasen der Kultivierung und der Barbarei einander wie Morgenund Abendröte ablösten. Mit den prophetischen Worten des französischen Schriftstellers und Philosophen François Fénelon von 1688: "Man muß nur Geduld haben, und wir werden nach einer lang dauernden Folge von Jahrhunderten wieder zu Zeitgenossen der Griechen und Römer." 14

In dieses zyklische, die Wiederkehr des Früheren beschwörende Bild paßte nach Fénelon bequem auch die Denkfigur einer "translatio imperii", einer Übertragung früherer kultureller und politischer Macht, so daß in Paris als dem neuesten Rom sich das Bild einer wiedererwachten, aber modernisierten, also qualitativ gesteigerten Antike wiedererkennen ließ. Was tatsächlich bedeutete, daß Paris die Zentrale der kulturellen Hegemonie in Europa werden wollte und wurde.

Zugleich aber überlagerte das Bild des kulturellen Kreislaufs, das lange vor 1789 mit dem Begriff der "Revolution", der zyklischen Umwälzung, verbunden war, ${ }^{15}$ eine andere nicht minder wirksame Geschichtsmetapher: nämlich das Bild eines linearen, eines unaufhaltsamen, vom Früheren immer weiter sich entfernenden Fortschreitens in die Zukunft. Eine prozeßhafte Vorstellung, die sich mit den teleologischen, also zielgerichteten Begriffen der Perfektion und der qualitativen Steigerung verschwistert hat. Die teleologische Zeitstruktur in der Bedeutung einer weltlichen Heilsgeschichte, nämlich einer Soteriologie der menschlichen Vernunft, wurde auch auf das Altertum übertragen. Und dieses erschien nun dem progressiven Blick als ein zeitlich und institutionell fortschreitender Weg zum Besseren, der von den scheinbar naiven und relativ unzivilisierten Anfängen der Griechen, über die römische Geschichte, die anschließende christliche Entwicklungsphase und die Überwindung eines kulturellen Zwischentiefs bis in die Gegenwart führte. Und diese das Frühere übertreffende Gegenwart ließ sich nun als Garant für eine verheißungsvolle, noch vollkommenere Zukunft deuten, weil sie fest an die Perfektibilität der Menschenwelt glaubte. Ein Optimismus, der den politischen Mächten, vor allem dem absoluten Staat, die Fähigkeit zuerkannte, die Zukunft unabhängig von der Nomothetik sakraler wie profaner Prophetien selbst in die Hände zu nehmen. ${ }^{16}$

Bedeutung hat "ancien" im Dictionnaire de l'Académie Française (T.I., Paris 1695, 24), während "antique" hier als Bezeichnung für "sehr alt" in Opposition zu "moderne" steht.

14. "Il ne faut qu'avoir patience; et par une longue suite de siècles, nous deviendrons les contemporains des Grecs et des Latins." F.Fénelon: Digressions sur les anciens e les modernes (1688). Zitat nach W.Krauss/H.Kortüm (Hg.): Antike und Moderne in der Literaturdiskussion des 18. Jahrhunderts, Berlin 1966, 67.

15. Vgl. zu den Ambiguitäten des Revolutionsbegriffs meinen Beitrag "Revolution und Mythos. Sieben Thesen zur Genesis und Geltung zweier Grundbegriffe historischen Denkens", in: D.Harth/J.Assmann (Hg.): Revolution und Mythos, Frankfurt/M. 1992, 9-35.

16. In diesem Kontext ist die Entscheidung der Modernisten für die zeitgenössischen Pionierleistungen der Technik und Naturwissenschaften bezeichnend. Diese entzogen sich nicht nur dem Vergleich mit den klassischen Idealen, sondern verweigerten sich auch-siehe das Beispiel der Schraube-dem Modell der Naturnachahmung. Damit konnte der Kampf gegen das alte Weltbild sich auf eine Ebene zurückziehen, die in ähnlicher Weise von Tradi- 


\section{Der Streit um den Vorrang der Moderne}

Das Besondere an dem Nebeneinander verschiedener Geschichtsmetaphern-der zyklischen Wiederkehr und der zielgerichteten Progression-liegt in der Vorbereitung eines veränderten, die Zukunftszeit einschließenden Zeitbewußtseins und in der Anerkennung perspektivisch gebundener Geschichtserwartungen. Geschichte ist danach nicht mehr allein die Darstellung des Vergangenen, sondern richtet sich auch auf die Zukunft: Die Vergangenheit wird an der Zukunft gemessen, der historische Blick zurück zum Leuchtturm der Modernisierung.

Je nach Standort in der Kultur der Gegenwart konnte man die Historie als Verfallsprozeß oder als Lernprozeß, als Tribunal oder als "magistra vitae", als Lehrmeisterin des Lebens, betrachten. ${ }^{17}$ Es ist daher auch kein Zufall, wenn Mitte des 18. Jahrhunderts dieser historische Perspektivismus als ein fundierendes Prinzip der Universalgeschichte anerkannt wird und zugleich mit dem Universalismus den Kulturrelativismus verschärft. ${ }^{18}$ Schon im Gelehrtenstreit über die Geltung Homers als Gesetzgeber der Dichtung, in der "Querelle d'Homère", tauchen zu Beginn des 18. Jahrhunderts die Leitbegriffe auf, die nach einer anderen als der autoritativen Rechtfertigung für die normative Vorbildlichkeit antiker Muster verlangen. So schreibt 1715 der englische Arzt und Schriftsteller Richard Blackmore in einem Essay über die epische Dichtung: "Unser Glaube [in Dichtungsfragen] darf nicht auf die Autorität der größten Meister vertrauen, sondern muß sich an zwingende Evidenz halten." Zur gleichen Zeit erläutert der französische Abbé Terrasson in einer kritischen Untersuchung über Homers Ilias, was unter dieser Evidenz zu verstehen ist: allein das, was sich vor dem Tribunal der Vernunft bewährt. ${ }^{19}$

Mit dem Kriterium der Vernunft aber wurde ein Prinzip ins Spiel gebracht, das als voraussetzungslos und insofern auch als geschichtslos galt, ein Prinzip also, von dessen scheinbar reinem Boden aus ein vollständig neues und vorurteilsloses, eben ein "modernes" Denken anheben konnte. Diderots Encyclopédie hat um die Mitte des 18. Jahrhunderts - übereinstimmend mit diesem universellen Vernunftbegriff-die Geburt der neuen Zeit auf den Beginn eines bis dahin unbekannten wissenschaftlichen Denkens datiert: die Astronomie des Kopernicus, die Physik eines Descartes und Newton bringen ein entzaubertes, ein rationales Weltbild hervor. Es sind demnach vor allem die Fortschritte in Wissenschaften und Technik, die als Anzeichen der Modernisierung allgemein anerkannt werden. ${ }^{20}$

tionsbeständen frei schien wie die tabula rasa der cartesischen Vernunft. Zum ideengeschichtlichen Hintergrund vgl. Hans Blumenberg: "'Nachahmung der Natur'. Zur Vorgeschichte der Idee des schöpferischen Menschen", in: Ders.: Wirklichkeiten, in denen wir leben, Stuttgart 1986, 55-103.

17. Zur Ablösung des magistra-Topos durch die historische Zeiterfahrung der Moderne vgl. Reinhart Koselleck: Vergangene Zukunft. Zur Semantik geschichtlicher Zeiten, Frankfurt/M. 1979, $38 \mathrm{ff}$.

18. Joh. Martin Chladenius: Allgemeine Geschichtswissenschaft, Leipzig 1752, 100.

19. R.Blackmore: Essay upon Epick Poetry (1716); Jean Terrasson: Dissertation critique sur l'Iliade (1715); beide zit. nach A.Owen Aldridge: "Ancients and Moderns in the Eighteenth Century", in: Dictionary of the History of Ideas, ed. P.P.Wiener, Bd. 1, New York 1973, 77.

20. Charles Perrault, der Wortführer der Modernen in der Querelle, hat ausdrücklich die methodische Konstruktion der Maschine als Fortschrittszeichen dem Prinzip der Naturnachahmung entgegengesetzt. Vgl. dazu Hans Robert Jauss: “Ästhetische Normen und geschichtli- 
In den Feldern positiven Wissens und analytischen Denkens hat die Moderne die Antike hinter sich gelassen; eine Zäsur wird bewußt, die zur Unterscheidung zwischen neuem und altem Denken anregt und zu jenen kritischen Bestandsaufnahmen führt, deren Monumente in den Enzyklopädien von Pierre Bayle bis d'Alembert/Diderot vorliegen. Diesem neuen Bewußtsein entspricht die erwähnte Bedeutung des Prädikats "antique": Es steht für das, was nicht mehr gebraucht wird, was aber als Beleg für den Fortschritt in die kritische Registratur gehört. Und dahin gehören vor allem-wie Pierre Bayle, der Verfasser des ersten mit traditionskritischem Anspruch geschriebenen Dictionnaire, überzeugt ist-die Irrtümer der Vergangenheit, ja die Irrtümer selbst der anerkanntesten Autoritäten. ${ }^{21}$ Mit einem Wort: die früheren Erkenntnisse werden Geschichte, sie werden-wie es später bei Hegel heißen wird-im "Tempel der Mnemosyne" hinterlegt. ${ }^{22}$ Wir sehen Descartes vor uns, wie er Aristoteles den Totenschein ausstellt, um an seine Stelle treten zu können.

Allein, was für Wissenschaften und Technik gilt, das soll in Literatur und Künsten anders sein, da in diesen nicht der messende und rechnende Verstand, sondern der abwägende Geschmack regiert. Das Geschmacksprädikat "moderne", so belehrt uns wiederum die Encyclopédie, steht nicht zu "ancien", sondern zum "mauvais goût", zum schlechten Geschmack der Gotik, in Opposition; andererseits aber sei nur die moderne Architektur schön zu nennen, die sich dem "goût de l'antique" annähere. ${ }^{23}$ Noch immer scheint hier-um die Mitte des 18. Jahrhunderts-jener klassizistische Code zu gelten, den das Zeitalter Ludwigs XIV. zur Norm erhoben hatte. Zwar interessierte sich niemand mehr für den Gebrauchswert der antiken Kunst- und Architekturelemente, aber genau das unterscheidet die neue Ästhetik vom Klassizismus.

Der damals übliche Kunstgeschmack legte ja nicht den Maßstab materieller Wertideen an noch bezog er die kanonischen Werke auf den Vergänglichkeitsmodus der historischen Zeit. Vielmehr urteilte dieser Geschmack, wie es bei einem zeitgenössischen Theoretiker hieß, "von der Schönheit der Kunstwerke nach ihrem Verhältnis mit der Natur". ${ }^{24}$ Bis weit ins 18. Jahrhundert bezeichnete der Naturbegriff etwas Konstantes, etwas, das vom Zahn der historischen Zeit unberührt schien. Und es hielt sich unglaublich lange unter Künstlern und Gelehrten der feste Gemeinplatz, daß Kunst und Literatur der Antike-trotz aller längst bemerkten zahlreichen lebensweltlichen Unterschiede mit der Gegenwartszeit-zu jener natürlichen Ordnung gehörten, hinter der die Idee einer vernunftgemäßen Vollkommenheit steht. Nicht die wilde, sondern die formal gebändigte, wie im französischen Garten domestizierte Natur befriedigte den rationalen Anspruch an eine gleichsam in die Zentralperspektive verlegte historische Wahrnehmung. Zugleich hat diese Beziehung der freien Künste und der politischmoralischen Normen auf ein gemeinsames, dahinter verborgenes Vernunftprinzip die

che Reflexion in der 'Querelle des Anciens et des Modernes'”, Einleitung zur Neuausgabe von Perraults Parallèle des Anciens et des Modernes [1688-97], (Parallèle des anciens et des modernes en ce qui regarde les arts et les sciences, mit einer einleitenden Abhandlung von H.R.Jauss und kunstgeschichtlichen Exkursen von M.Imdahl, Theorie und Geschichte der Literatur und der schönen Künste. Texte und Abhandlungen, 2), München 1964, 49.

21. Vgl. Ernst Cassirer: Die Philosophie der Aufklärung, Tübingen ${ }^{3} 1973,269 \mathrm{ff}$.

22. G.W.F.Hegel: Die Vernunft in der Geschichte, hg. von J.Hoffmeister, Hamburg ${ }^{5} 1970,4$.

23. Encyclopédie, T. X, Paris $1765,601$.

24. Johann Adolf Schlegel: "Abhandlungen", in: Charles Batteux: Einschränkung der schönen Künste auf Einen einzigen Grundsatz, Leipzig 21759, 253. 
Kooperation zwischen den Vertretern des modernen Denkens, den "philosophes", und dem aufgeklärten Adel erleichtert.

Aufgrund dieser Prämisse erschienen die antiken Kunstwerke dem Auge der Traditionalisten als etwas Formvollendetes, und ihre Mustergültigkeit für den eigenen kulturellen Kosmos wurde im System der artistischen Logik immer wieder aufs neue umgeschrieben. Ein Verfahren, das Traditionalisten und Modernisten gemeinsam war, auch wenn sie über den Inhalt des antiken Kanons im Streit lagen. Ohnehin gingen bald beide Parteien von der Vollendung der Kunst-Antike im Sinne einer in Distanz gebrachten Abgeschlossenheit aus. Das war zugleich eine notwendige Bedingung für den Vergleich zwischen Jetzt und Damals und für den Entwurf eines kulturellen Codes, der die Antike in ihrer Abgeschlossenheit als ein Modell anerkannte, ein Modell, das es zu studieren galt und mit dem man in Konkurrenz treten konnte, ohne das Neue verleugnen zu müssen..$^{25}$

Diese Modellkonstruktion der Kunst-Antike verlangte nach dauernd zu verbessernder Evidenz im Sinne der historisch-philologischen Kritik. ${ }^{26}$ So daß der Streit um die Geltung des klassischen Kanons-und das gilt für ganz Europa-der Produktion gelehrten Wissens über die Alte Welt eine enorme Konjunktur verschaffte. An der zugleich damit einsetzenden methodischen Objektivierung der Antike als einer eigenständigen und einheitlichen, ja wie ein Organismus als Ganzheit zu betrachtenden Lebensform konnte sich dann der Gedanke festmachen, daß die eigene Zeit einen höheren Grad an kultureller Komplexität aufzuweisen hatte und schon allein dadurch sich von der Tradition klar unterschied. So wurde die für den Klassizismus repräsentative Figur des "poeta doctus" auf ein Lernpensum verpflichtet, das dieser beobachteten kulturellen Differenzierung und ihrer historischen Vielfalt Rechnung trug. Um ein frühes englisches Beispiel zu zitieren: Der Publizist und Dichter John Dryden verlangte im Sinne rhetorischer Schulvorschriften vom epischen Dichter zugleich Erfindungsund Urteilsvermögen. Aber das allein genügte ihm nicht. Er forderte darüber hinaus vom Dichter "die Kenntnis der freien Künste und Wissenschaften, besonders aber der Moralphilosophie, der Mathematik, der Geographie und der Geschichte". ${ }^{27}$

Das sind Forderungen, die den Abstand zwischen Homer und dem Dichter der Moderne unübersehbar hervortreten lassen. Zwei Generationen später aber wurde dieses Argument überraschenderweise umgekehrt: Homer wurde nun zum Paradebeispiel für eine ohne Gelehrsamkeit auskommende, für eine naturgemäße bzw. naive Poesie, für die Genie-Dichtung. ${ }^{28}$ In beiden Fällen aber war der zeitliche Abstand die notwendige Bedingung für den Ausbau des antiken Kanons zu einem maßstabsetzenden Modell.

Der kulturelle Imperativ des "esprit classique" aber lautet seitdem: Die Antike muß studiert werden! Winckelmann, Diderot und viele andere haben diesen Imperativ

25. S. zu dieser Art der Modernisierung des Alten Luc Ferry: Der Mensch als Ästhet. Die Erfindung des Geschmacks im Zeitalter der Demokratie, Stuttgart 1992, 54ff.

26. Vgl. zu diesem gelehrten Aneignungsprozeß Arnaldo Momigliano: Wege in die Alte Welt, Berlin 1991. Über die Vorbereitung der ästhetisierenden Kunstbetrachtung durch die gelehrte Archäologie: Luigi Beschi: "La scoperta dell'arte greca", in: S.Settis: Memoria dell'antico nell'arte italiana, III, Torino 1986, 293-372.

27. Zitat nach Aldridge [Anm.19], 78.

28. Vgl. dazu Jochen Schmidt: Die Geschichte des Genie-Gedankens 1750-1945. I: Von der Aufklärung bis zum Idealismus, Darmstadt 1985, 27 u.ö. 
propagiert und befolgt. In welchem Maße dieses Gebot den übergreifenden Code eines europäischen Kulturbewußtseins ausgebildet hat, das gehört zur Geschichtsschreibung der pädagogischen Ideen und Bildungsinstitutionen. Jedenfalls überlebte es bis weit ins 19. Jahrhundert im Gymnasium und in der Universität. ${ }^{29}$ Und es ist verantwortlich für jene Konkurrenz zwischen den Normen einer wissenschaftlich-technischen Kultur einerseits und einer klassisch-literarischen Kultur andererseits. Ein Konkurrenzverhältnis, das zuerst im Zeitalter der "Querelle" bemerkt worden ist und in bildungspolitischen Fragen die Auseinandersetzung zwischen Modernisten und Traditionalisten nie wieder hat zur Ruhe kommen lassen.

Dieser Streit zwischen einerseits durch Alter und Tradition beglaubigten und andererseits zukunftsbezogenen Normen und Wertideen macht aber nur deutlich, wie sehr der Gedanke permanenten Lernens zum Selbstverständnis der Moderne gehört. Denn je radikaler die evolutionäre Struktur der Moderne als ein unendlicher Lernprozeß verteidigt wurde, um so klarer trat das Bild einer vollendeten, aber ein für allemal abgeschlossenen Antike hervor. Dies voraussetzend hat der französische Schriftsteller Voltaire die Universalgeschichte der europäischen Kultur in vier Perioden eingeteilt: 1. das Zeitalter Alexanders oder des Perikles, dessen Kultur in Opposition zur Barbarei der übrigen Welt stand, weshalb sie sich als Urszene der Kulturentstehung betrachten ließ; 2. das Zeitalter Cäsars und des Augustus; 3. die Epoche der Medicidie Renaissance-und schließlich. 4. das "Siècle de Louis XIV". Was diese Zeitalter im Sinne einer evolutionären Wertsteigerung miteinander verbindet, beschreibt Voltaire mit folgenden Worten: "In diesen vier glücklichen Zeitaltern wurden die Künste perfektioniert. Sie stehen seitdem im Dienst der Größe des menschlichen Geistes und sind ein vorbildliches Muster für die Nachwelt (l'exemple de la postérité)."30

In dieser die Geschichte der Künste und des Geistes zur Deckung bringenden Beobachtung zählt nicht mehr die Antithese oder Konkurrenz zwischen Antike und Moderne. Was zählt, das ist allein ihr Beitrag zu jenem Lernprozeß des "esprit humain", der in der vierten Epoche einen für alle kommenden Zeiten exemplarischen Höhepunkt erreicht hat. Damit ist das Zeitalter des Sonnenkönigs und des absoluten Staates an jene Stelle getreten, die in der "Querelle" auf Seiten der Traditionalisten noch die Antike eingenommen hatte. Dem kulturellen Zentralismus dieser neuen Blütezeit, der die Künstler und Architekten Ludwigs XIV. in Paris und Versailles ein ästhetisch wahrnehmbares Gesicht verliehen haben, ${ }^{31}$ entsprach eine intellektuelle Zentralperspektive, die alle kulturellen Erscheinungen auf den einzigen Fluchtpunkt des Vernunftprinzips bezog. "La raison humaine en général s'est perfectionnée", schrieb Voltaire schwärmerisch über die Vervollkommnungen der menschlichen Vernunft, und er fügte sogleich hinzu: "Das hat zu einer allgemeinen Umwälzung (une révolution générale) in unseren Künsten, in unserem geistigen Leben, unseren Sitten wie auch in unserer Regierungsform geführt." 32

29. Zu den philologiehistorischen Grundzügen dieser Entwicklung vgl. Rudolf Pfeiffer: History of Classical Scholarship from 1300 to 1850, Oxford 1976, 183ff. (Id., Die Klassische Philologie von Petrarca bis Mommsen, München 1982, 207ff.)

30. Voltaire: Le siècle de Louis XIV, I, Paris 1966, 35.

31. Vgl. Francis Haskell \& Nicholas Penny: Taste and the Antique. The Lure of Classical Sculpture 1500-1900, New Haven/London 1981, 36ff.

32. Voltaire [Anm.30], 36. 


\section{Die Antike als Denkbild der Moderne}

Man muß nur die Perspektive zurechtrücken, um zu erkennen, daß es diese von Voltaire vertretene, unter der Vormundschaft des "esprit classique" ausgebildete Wahrnehmungsweise war, die jener Ästhetisierung und Universalisierung der Kunst-Antike den Boden bereitet hat, als deren Urheber der deutsche Gelehrte und Kunsthistoriker Johann Joachim Winckelmann anzusehen ist. Mit Winckelmanns Name ist jene Entwicklung verbunden, die im deutschen Idealismus die Modernisierung der Antike vollendet, indem sie deren Idee-wohlgemerkt: nicht ihre historische Realität-als einen tragenden Baustein ins Systemgebäude der geschichtsphilosophisch fundierten Ästhetik einfügt. Dieses geht aus dem Protest gegen die Hegemonie des "esprit classique" hervor. ${ }^{33}$ Insofern verdankt die neue Konstruktion ihre entscheidenden Impulse dem übermächtigen kulturellen Code des an Rom geschulten französischen Klassizismus, den sie studiert und bekämpft hat. Das Resultat, die geschichtsphilosophische Konstruktion der griechischen Antike, setzt voraus, daß sowohl auf der Makro- wie auf der Mikroebene das scheinbar einheitliche Geschichtsbild der Antike sich sowohl in wertender als auch in zeitlicher Perspektive in mehrere Facetten zerlegt hat. ${ }^{34} \mathrm{Zu}$ erinnern ist hier auch an die ideologische Opposition von griechischer und römischer Antike, an die Aufgliederung in Stilperioden und an jenes Modelldenken, das die nach Maßgabe des jeweiligen Anteils an Rationalität zu unterscheidenden Vergangenheiten in den Evolutionsprozeß der Vernunft eingebunden hat.

Im deutschen Sprachraum blieb die Diskussion über den Vorzug von Antike oder Moderne zunächst akademisch. ${ }^{35}$ Sie war weitgehend von theologischprotestantischen Ideen geprägt. Das bedeutendste Resümée erschien 1735 in Leipzig bezeichnenderweise noch in der lateinischen Gelehrtensprache: Georg Heinrich Ayrers Dissertatio de comparatione eruditionis antiquae et recentioris. Und wenn von Fortschritten im Sinne der Modernisierung die Rede war, dann verwiesen die gelehrten Autoren lieber auf Luthers Reformation als auf die katholische Renaissance oder gar auf die Ära des französischen Sonnenkönigs. Was neu war, erschien daher nicht im Licht weltlicher Fortschritte, sondern als Reform religiöser Inhalte und Institutionen. Damit war aber das Vernunftprinzip eines neu anhebenden Denkens schwerlich zu vereinbaren. Wer das moderne Denken favorisierte, war daher wie Lessing gezwungen, streng zwischen Vernunftfortschritt und symbolischer, wohl auch moralischer Zuständigkeit der Offenbarung zu unterscheiden. ${ }^{36}$ Im Reich der Künste und Poesie ließ sichwie Lessing wußte-die "Schule der Alten" auch ohne Klassizismus nutzen. Es kam nur darauf an, ihre Muster und Kriterien zu "modernisieren". Und das warf die Frage nach dem auf, was zum Kanon dieses Studiums gehören sollte. Lessing fiel die Entscheidung leicht: Die dramatische Schule der Alten lag für ihn dort, wo die griechische

33. Zu Winckelmanns Kenntnis französischer Quellen s. Martin Fontius: Winckelmann und die französische Aufklärung, Berlin 1968.

34. Das Interesse der französischen Intellektuellen für die griechische Antike war vor allem politisch und rhetorisch motiviert; vgl. R.Zuber: "France 1640-1790", in: K.J.Dover (Hg.): Perceptions of the Ancient Greeks, Oxford 1992, 147-169.

35. Vgl. Peter K.Kapitza: Ein bürgerlicher Krieg in der gelehrten Welt. Zur Geschichte der Querelle des Anciens et des Modernes in Deutschland, München 1981.

36. Vgl. die entsprechenden Kapitel in meinem Buch G.E.Lessing oder die Paradoxien der Selbsterkenntnis, München 1993. 
Aufklärung, d.h. die sokratische Philosophie mit ihrem "Erkenne Dich selbst!", ihre mächtig weiterwirkenden Spuren hinterlassen hatte: in den attischen Irrtumstragödien des Sophokles und Euripides.

Für Winckelmann, mit dem Lessing sich gründlich im Laokoon auseinandersetzte, lagen die Dinge anders. Der Kulturhistoriker Egon Friedell hat Winckelmann nachgesagt, er habe die griechische Antike schlicht erfunden, ja sie in eine blutleere Attrappe verwandelt. Wenn man "Erfindung" in das lateinische Wort "inventio" rückübersetzt und nicht als substanzlose Phantasterei wie Friedell versteht, so ist dem durchaus zuzustimmen. Denn "inventio" bezeichnet eine heuristische, methodisch vorgehende Untersuchungsart, die gerade nicht frei phantasierend erfindet, sondern sich an gegebene Materialien und Quellen hält. Winckelmann hat eine Qualität an der griechischen Plastik des 5. vorchristlichen Jahrhunderts entdeckt, die schon in ihrer Entstehungszeit, also von den Griechen selber, als eine Neuerung, als ein Zeichen der Ablösung von Traditionen begriffen worden ist. Bevor ich das genauer erläutere, möchte ich jedoch einige Randbedingungen skizzieren, die für das Verständnis dieser "inventio" und ihre nachhaltige Wirkung im Feld der allgemeinen und der Kunst-Geschichte notwendig sind.

$\mathrm{Zu}$ der mit Winckelmann einsetzenden Ästhetisierung der griechischen Antike gehören einige Voraussetzungen, die den allgemeinen Wandel der Kunstauffassung im späten 18. Jahrhundert betreffen. Auf der Ebene der materiellen Forschung hat die Unterscheidung zwischen römischer und griechischer Bildkunst überhaupt erst in diesem Jahrhundert Fortschritte gemacht. ${ }^{37}$ Bis dahin wurde das Bild der antiken Kunst weitgehend von literarischen Interessen beherrscht: Antiquare, Philologen und Historiographen sahen in der Bildkunst vor allem das Illustrationsmaterial für vergangene Ereignisse und philologisch-historische Streitfragen.

Mit der Rehabilitierung der Sinnlichkeit und der Einbildungskraft durch Sensualismus und Empirismus setzt eine Umwertung ein. So wird die antike Mythologie kräftig als ein eigenständiger, auf die schöpferische Imagination verweisender Bereich der Bildproduktion aufgewertet. Sie ist nicht mehr allein, was an der HomerDiskussion zu belegen wäre, die bildliche Einkleidung allegorisch $\mathrm{zu}$ enträtselnder Ideen oder Ausdruck eines ästhetischen Manierismus. ${ }^{38}$ Sie wird vielmehr als Kriterium der imaginativen Sprachkunst aufgewertet. Hier sind symbolische Formen gefragt, die sich gerade nicht in Bekanntes rückübersetzen lassen, sondern zur unendlichen Auslegung herausfordern. Eine späte Bemerkung Goethes bringt diese ästhetisierende Wiederanknüpfung an das "unendlich Motivbare" der antiken Symbole auf den Begriff: "Die griechische Mythologie, sonst ein Wirrwarr, ist nur als Entwickelung der möglichen Kunstmotive, die in einem Gegenstande lagen, anzusehen" ${ }^{39}$

Die einer solchen Auffassung zugrundeliegende Bildtheorie, die im deutsch-

37. Vgl. Orietta Rossi Pinelli: "Chirurgia della memoria: scultura antica e restauri storici", in: Settis [Anm.26], 181-250.

38. Zur neueren Geschichte der Homer-Kritik vgl. Glenn Most: "The Second Homeric Renaissance. Allegories and Genius in Early Modern Poetics", in: P.Murray: Genius. The History of an Idea, Oxford 1989, 54-75.

39. Goethe: Berliner Ausgabe, XIX, Berlin 1973, 378. J.W.Goethe: Gedenkausgabe der Werke, Briefe und Gespräche, XXII, Zürich 1949, 566. Zur zeitkritischen, die modernen 'Anfechtungen' aushaltenden Funktion dieses Denkens vgl. Bernhard Buschendorf: Goethes mythische Denkform. Zur Ikonographie der "Wahlverwandtschaften", Frankfurt/M. 1986, $29 \mathrm{ff}$. 
sprachigen Raum von den Schweizern Bodmer und Breitinger und in England von Edmund Burke vorbereitet worden ist, spricht dem Mythologem eine semantische Kraft zu, die dem logischen Vermögen, nämlich dem begrifflich operierenden Verstand, unzugänglich bleibt. Als "Sprache der Phantasie" soll die Mythologie eine evokative Kraft entfalten, die sich nicht der Rationalität, sondern nur der Wahrnehmung und Erfahrung, oder mit einem Schlüsselwort Herders zu sprechen: der "Empfindung" des Betrachters erschließt. ${ }^{40}$

Diese Entdeckung erlaubte es, der vom Rationalismus geübten Reduktion der Phänomene auf das zentrale Vernunftprinzip eine ganz andere Argumentation entgegenzusetzen. Ihre theoretische Grundlegung findet diese neue Position in der ersten systematischen Lehrschrift der Kunstphilosophie, die Alexander Gottlieb Baumgarten-noch in lateinischer Sprache-1750/58 unter dem Titel Aesthetica veröffentlicht hat. Ungeachtet ihrer rhetorischen Form und ihrer rationalistisch-schulphilosophischen Methode setzt diese Schrift sich dafür ein, dem herrschenden Muster rationaler Erkenntnis eine andere Erkenntnisweise an die Seite zu stellen. Eine Erkenntnisweise, die nicht allein den logischen Zusammenhalt der Welt, sondern vor allem die Erfahrung des anschauenden Subjekts in Rechnung stellt. Die sinnliche bzw. ästhetische Erkenntnis reduziert das Wahrgenommene nicht im Sinne der vergleichenden, auf gemeinsame Merkmale, auf Klassifikation, zielenden Abstraktion. Im Gegenteil: sie erfährt die Dinge in ihrer Einzigartigkeit, in ihrer unvergleichlichen, unreduzierbaren Fülle (plenitudo) und d.h.: in der Art und Weise, wie sie erscheinen. Als Erscheinung (phaenomenon) ist der Anschauungsgegenstand ein Produkt der ästhetischen Erkenntnis und insofern abhängig vom perspektivischen Standort des anschauenden Subjekts. ${ }^{41}$

Winckelmanns Bild der griechischen Antike ist im Kontext dieser neuen, der ästhetischen Anschauungsform zu deuten, auch wenn er Baumgartens Lehrschrift nicht gekannt haben sollte. Denn auch Winckelmann konzentriert sich auf das einzelne Bildwerk, und er interpretiert es in der Weise seines Erscheinens als Ausdruck einer nur gleichnishaft zu umschreibenden Fülle. Deren Schönheitsmaß, das Winckelmann zur Norm erhebt, lautet: Einheit in der Mannigfaltigkeit. Auch dies ist eine Kategorie des Maßes, der ausgeglichenen Spannung. Die vollkommene griechische Plastik, schreibt Winckelmann, ist "wie die Einheit der Fläche des Meeres, welche in einiger Weite eben und stille wie ein Spiegel erscheint [!], ob es gleich alle Zeit in Bewegung ist und Wogen wälzt". ${ }^{42}$

Das Maß der Vollendung und Schönheit beruht also auf einer prekären, auf einer augenblickshaften Balance, die den Extremen der zeitresistenten Starre und der zeitlichen Bewegung nicht abgeschaut, sondern geradezu abgetrotzt ist. Deshalb kann Winckelmann sagen, daß die griechischen Künstler nicht die äußerliche, sondern "eine im Verstand entworfene geistige Natur" zum "Urbild" hatten. ${ }^{43}$ Nicht die Natur, wie

40. Vgl. z.B. Herders frühe "Fragmente einer Abhandlung über die Ode" [1765]. in: Sämmtliche Werke, hg. von B.Suphan, XXXII, Berlin 1899, 61-85. Karl Phillip Moritz beginnt seine Götterlehre oder Mythologische Dichtungen der Alten von 1791 mit dem Satz: "Die mythologischen Dichtungen müssen als eine Sprache der Phantasie betrachtet werden.", in Ders.: Schriften zur Ästhetik und Poetik, ed. H.J.Schrimpf, Tübingen 1962, 195.

41. Alexander Gottlieb Baumgarten: Theoretische Ästhetik. Die grundlegenden Abschnitte aus der "Aesthetica" (1750/58), übers. u. hg. von H.R.Schweizer, Hamburg 1983, Einleitung des Hg., p. XIII.

42. Johann Joachim Winckelmann: Geschichte der Kunst des Altertums [1764], Darmstadt 1982, 152.

43. J.J.Winckelmann: Ausgewählte Schriften und Briefe, hg. von W.Rehm, Wiesbaden 1948, 9. 
sie ist, sondern ihre Idee, wobei zu erinnern ist, daß das griechische Wort "idea" nichts anderes meint als ein geistig wahrnehmbares Bild. Damit verändert Winckelmann nicht nur den Sinn der traditionellen Natur-Nachahmungs-Formel, er gibt auch der ästhetischen Mimesis, der Kunst der Darstellung, eine neue Bedeutung, die bis heute Bestand haben kann. Vorausgesetzt, wir sind bereit, unter dem Begriff der Mimesis die Fähigkeit der Künste und Poesie anzuerkennen, das Unsichtbare sichtbar, das Unsagbare sagbar, das Unhörbare hörbar zu machen, es also in seiner Fülle zur Erscheinung zu bringen und nicht auf konventionelle Wahrnehmungs- und Bezeichnungsmuster zu reduzieren.

Winckelmanns "Natur" ist-das gilt es zu bedenken-kein Beschreibungs-, sondern ein Reflexionsbegriff. Will sagen: ein Begriff, der sich nicht auf das äußerlich wahrnehmbare Naturbild bezieht-auf die Oberfläche der Landschaft oder eines organischen Körpers, sondern auf die in der Natur wirkende schöpferische Kraft. Er überträgt ihn auf die Antike, und zwar auf die ganze nach ihm so benannte "klassische" Epoche der griechischen Plastik des 5. vorchristlichen Jahrhunderts. Ein außerordentlich gut gewähltes Paradigma, hervorragend geeignet, um dem normativen Antikebild des französischen Klassizismus entgegenzutreten. Denn die ältere griechische Plastik des 6. vorchristlichen Jahrhunderts war in ähnlicher Weise normativ auf die Befolgung traditioneller Schemata festgelegt wie der französische Klassizismus und dazu noch Ausdruck einer aristokratischen Lebensordnung, in der das unveränderliche Gesetz althergebrachter Lebensformen mehr Geltung besaß als die Freiheit der Innovation und die Idee der Veränderung. ${ }^{44}$ Mit der Wahl der spannungsgeladenen, die Archaik durch die Bewegungsfigur überwindenden Plastik wendet sich Winckelmann jener Epoche $\mathrm{zu}$, in der die Griechen die Geschichtsschreibung entdeckt haben und vom politischen Nomos zur Demokratie übergingen. Unter dieser Voraussetzung kann Winckelmanns "Erfindung" der klassischen Antike-in der austauschbaren Bedeutung der Skulptur und der Epoche ${ }^{45}$ _durchaus als eine kongeniale Leistung angesehen werden.

Und doch ist nicht zu übersehen, daß diese "Erfindung" unter dem Zeichen jener Modernisierung steht, die ich eingangs mit kultursoziologischen Begriffen umschrieben habe. Denn Winckelmanns Entwurf ist nicht aus historisch-antiquarischen Interessen entstanden. Er folgte vielmehr einer geschichtsphilosophischen Intuition, die in der Kunstphilosophie des Idealismus aufgegriffen und zu einem Phänomen ausgebildet worden ist, das keine Ähnlichkeit mit der historischen Antike hatte und diese auch gar nicht suchte. Grund war die Erfahrung eines Mangels, eine Erfahrung, die von vielen deutschen Autoren formuliert worden ist: das Fehlen einer kulturellen, einer identitätsstiftenden Einheit, ein Mangel, den die deutschen Intellektuellen des ausgehenden

44. Vgl. Tonio Hölscher: "Tradition und Geschichte. Zwei Typen der Vergangenheit am Beispiel der griechischen Kunst", in: J.Assmann/T.Hölscher (Hg.): Kultur und Gedächtnis, Frankfurt/ M. 1988, 115-149. Zur dialektischen, die Spannung zwischen Starre und Bewegung aushaltenden Tendenz in Winckelmanns Ästhetik vgl. Peter Szondi: Poetik und Geschichtsphilosophie I: Antike und Moderne in der Ästhetik der Goethezeit. Hegels Lehre von der Dichtung, hg. von S.Metz/H.-H.Hildebrandt, Frankfurt/M. 1974, 43ff.

45. In der Allgemeinen Encyclopädie der Wissenschaften und Künste, von Ersch \& Gruber (4.Theil, 1820, 300ff.) schreibt Bouterwek, erst das Studium des "Antiken im ästhetischen und artistischen Sinne" habe "das Moderne" hervorgebracht. Noch im Deutschen Wörterbuch der Brüder Grimm von 1854 (Bd.1, 500) finden sich unter dem Lemma "Antike" nur die aufs Kunstwerk verweisenden Primär-Erläuterungen: "opus antiquum, artis opus". 
18. Jahrhunderts gern mit der Metapher der "Zerrissenheit" in Verbindung gebracht haben. ${ }^{46}$ Die auf die klassische Antike übertragene Einheit von Natur, Geist und Kunst soll—schrieb Winckelmann—dem modernen Künstler zur Regel werden: "Die Begriffe des Ganzen, des Vollkommenen in der Natur des Altertums werden die Begriffe des Geteilten in unserer Natur bei ihm läutern und sinnlicher machen: er wird bei der Entdeckung der Schönheiten derselben diese mit dem vollkommenen Schönen zu verbinden wissen, und durch die Hilfe der ihm beständig gegenwärtigen erhabenen Formen wird er sich selbst eine Regel werden." 47

Der Umgang mit der Antike ist, wie diese Formulierungen zeigen, einerseits begriffliche Arbeit, andererseits aber begriffslose Anschauung. Wo beides zusammenkommt, wo die Synthese von sinnlicher Anschauung und Reflexion gelungen ist, dort entsteht ein schöpferisches Selbstbewußtsein im Künstler, dort wird er sich selbst zur Regel.

In ähnlicher Weise argumentiert Winckelmann in seinen Aussagen über den Begriff des Schönen. Denn das Erhabene der Formen-und das ist ein Kriterium vollkommener Schönheit — entzieht sich nach seinem Verständnis der rationalen Identifizierung, vergleichbar der Resistenz der phänomenalen Fülle gegenüber den Begriffen, wovon Baumgartens Ästhetik sprach. Winckelmann bemüht daher die Gleichnissprache der christlichen Mystik, um die schöpferische Potenz anzudeuten, die sich in der Anschauung des in der antiken Plastik verkörperten erhabenen Schönen dem Betrachter mitteilen soll: "Schönheit ist wie ein aus der Materie durchs Feuer gezogener Geist, welcher sich sucht ein Geschöpf zu zeugen nach dem Ebenbilde der in dem Verstande der Gottheit entworfenen ersten vernünftigen Kreatur." 48

Nach diesen Worten ist es der Geist der Schönheit, der noch einmal-im Zeitalter des Mangels und der Zerrissenheit-das Bild jenes Menschen zu formen versteht, der vor dem Sündenfall, d.h.: vor der Zeit der Teilung, existiert haben soll. Dieses Bildnis ist Individuum, ein unteilbares Ganzes, und zugleich-durch den objektiven Ausdruck seiner erhaben-schönen Form-Repräsentant eines Allgemeinen, nämlich der Menschheit im Zustand ihrer Erlösung von der Endlichkeit der Zeit und vom Verlust der Ganzheit. ${ }^{49}$

Antike und Moderne treten in Winckelmanns Konzeption in ein Verhältnis der theoretischen Vermittlung, das mit der historischen Epochengliederung nichts mehr zu tun hat. Der klassischen Antike wird vielmehr als eines Symbols für die Überwindung der in der Gegenwartszeit erfahrenen Mängel gedacht. Sie ist das Denkbild der mit sich selbst versöhnten Moderne. Dieser Konstruktion liegt die Bewegungsformel für jenen geschichtsphilosophischen Dreischritt zugrunde, der in der idealistischen und romantischen Kunstphilosophie zwischen (1) ursprünglicher, vorreflexiver Einheit, (2) Zerfall durch Widerstreit in der Gegenwart und (3) künftiger Wiederherstellung im Sinne einer vom philosophischen Gedanken ausgehenden eschatologischen Utopie unterscheidet. Der christlich-

46. Vgl. meinen Essay "Zerrissenheit. Der deutsche Idealismus und die Suche nach kultureller Identität", in: Assmann/Hölscher [Anm.44], 220-240.

47. Ausgewählte Schriften [Anm.43], 13f.

48. a.a.O., 124 .

49. Die intendierte semantische Ähnlichkeit zwischen dem Ganzen einer Kulturnation, eines ästhetischen Werkes und universeller Menschheitsrepräsentanz faßt, soweit hier Präzision überhaupt möglich, am genauesten Wilhelm von Humboldt in seinen verschiedenen Studien über die identitätsbildende Funktion der klassischen Bildung: Schriften zur Altertumskunde und Ästhetik, Werke II, hg. von A.Flitner/K.Giel, Darmstadt 1961. 
theologische Hintergrund, der Dreischritt Paradies—Sündenfall—Erlösung, ist hier ebensowenig zu übersehen wie in Winckelmanns Synthese des biblischen Schöpfungsmythos mit der antiken Ethik des zwischen Extremen vermittelnden Maßes.

Was aus diesem Prozeß einer Ästhetisierung der griechischen Antike hervorging, an dem sich fast alle deutschprachigen Schriftsteller beteiligten, das war ein Kulturideal, in dessen reflexiv hervorgetriebenem Gegenbild die moderne Kultur-als nationales System verstanden-sich spiegeln konnte, um-paradox genug-ihre Besonderheit, ihre grundsätzliche Andersartigkeit, wahrzunehmen. Andersartigkeit insbesondere im Vergleich mit dem kulturellen Code der französischen Nachbargesellschaft. Denn eine Spiegelung zeigt sich überall dort, wo die Epoche der griechischen Antike als ein Kunstwerk angesehen wird, dessen Form die ausgehaltene Spannung zwischen den Extremen der Starre einerseits und der Bewegung andererseits zum Ausdruck bringt. In dieser Sicht wird die von Winckelmann für die ganze "klassische" Antike symbolisch eingesetzte skulptierte menschliche Gestalt lesbar als Emblem der zwischen Beharrung und Aufbruch vermittelnden Stellung des modernen Intellektuellen. Eine Stellung, die für das Selbstverständnis der deutschen in viel höherem Maß als für die französischen Intellektuellen kennzeichnend war und je verschiedene Formen der Traditionskritik in der einen wie der anderen Gesellschaft hervorgebracht hat.

Ende des 18. Jahrhunderts-anders als zur Zeit der französischen "Querelle" - haben die deutschen Intellektuellen nicht mehr den Wettbewerb mit der Antike gesucht. Die auf Kunst, Poesie und Politik der Griechen projizierte Totalität in der Bedeutung einer kollektiven, als Kulturnation gedachten "natürlichen" Lebensform gehörte, wie Friedrich Schiller immer wieder bemerkte, jener symbolischen Ordnung des ästhetischen Scheins an, von der aus sich der trügerische Schein einer ohne Verluste fortwurstelnden Moderne kritisch betrachten und-im subjektiven Raum der ästhetischen Erfahrungüberwinden ließ. "Das Vergangene ist nicht vergangen," schreibt Karl Philipp Moritz, "so lange es noch in jeder neu aufkeimenden Einbildungskraft sich wieder verjüngen muß." Im "Bild" des Altertums, das die Imagination erzeugt, wird die Komplexität der Moderne (das "Verwickelte") überwunden, und vor dem inneren Auge erscheint das Ideal der antiken Welt als universell gültige Chiffre der "Menschheit" ${ }^{50}$

Vor dem Hintergrund der theoretisch und ästhetisch konstruierten virtuellen Realität der Antike hob sich nun auch die Moderne als genauer zu bestimmende Wirklichkeit ab. Schiller beschrieb "unsere unpoetischen Tage" mit Worten, deren Kern Kampf und Desorientierung bedeutet: Vereinzelung, Trennung, Absonderung, Antagonismus usf. ${ }^{51}$ Die Moderne besitzt, wie Friedrich Schlegel in seinem Essay Über das Studium der griechischen Poesie andeutet, die Einheit des Labyrinths, entbehrt also eines leicht zugänglichen und verbindlich ordnenden Zentrums. ${ }^{52}$ Die Zeitstruktur der Moderne ist offen wie der ewig suchende Gang durch den verschlungenen Bau des Labyrinths. Und gerade um dieser Offenheit willen bedarf der Künstler und Poet, der in dieser Konzeption als ein Leitbild des modernen Weltbewohners und als Produzent zunkunftsweisender Symbole-der sog. "neuen Mythologie" —erscheint, eines Ariad-

50. Moritz [Anm.40], 106f. Vgl. auch die Nr. 6 von Schillers Briefen über die ästhetische Erziehung des Menschen.

51. F.Schiller, Sämtliche Werke, Säkular-Ausgabe, Stuttgart/Berlin [o.J.], XII, 22, 228; XVI, 227.Zu Schillers ethnozentrischer Relativierung des französischen Klassizismus vgl. u. a. seine Gedichte "An Goethe" (1800) und "Die Antiken zu Paris" (1803).

52. F.Schlegel: Schriften zur Literatur, hg. von W.Rasch, München 1972, 156. 
nefadens. Diesen soll ihm noch einmal die "verstehende", und d.h.: die hermeneutisch aneignende Anschauung der "reinen Griechheit", in die Hand geben. ${ }^{53}$ "Reine Griechheit": das war Schlegels abstrahierender Einheitstitel für jene Universalien, die der deutsche Intellektuelle als Spitzenwerte seines eigenen kulturellen Codes hochschätzen sollte. Mit Schlegels Worten: "sittliche Fülle, freie Gesetzmäßigkeit, liberale Humanität". Und mit unverkennbar nationalem Pathos faßt er zusammen: "In Deutschland, und nur in Deutschland, hat die Ästhetik und das Studium der Griechen eine Höhe erreicht, welche eine gänzliche Umbildung der Dichtkunst und des Geschmacks notwendig zur Folge haben muß." 54

Die Historisierung der Antike, die in Herders Forderung, sie nicht nachzuahmen, sondern vielmehr zu erklären, Programm geworden ist, hat ihre normative Geltung als Vergleichs- und Gegenmodell zur Moderne relativiert. ${ }^{55}$ Die Ästhetisierung soll den so angeschlagenen Grund wiederherstellen, auf dessen schmaler intellektueller Basis die antike griechische Kultur als Denkbild der mit sich selbst, nämlich mit ihren utopischen Idealen versöhnten Moderne zu errichten ist. Der kulturelle Universalismus, der die Welt der Griechen zum Menschheitsideal erhebt, stößt jedoch an Grenzen, die den Anspruch auf Allgemeingültigkeit wiederum relativieren. Denn es ist nicht zu übersehen: Die deutschen Intellektuellen im hier betrachteten Zeitraum kämpfen mit dem Instrument der klassischen Bildung für einen nationalen Mythos, der nicht nur mit dem französischen Universalanspruch konkurriert, sondern ihn zu überbieten sucht, indem er die dynamischen Fortschritte der französischen Moderne mit dem statuarischen Idealbild der griechischen Antike zusammenzwingt. ${ }^{56}$ Ein Traum, der rasch zerstört wird, da die Revolutionskriege bald die kulturellen Ansprüche der einen wie anderen Seite in die Sprache der Gewalt übersetzen. An die Stelle der universalistischen antiken Denkbilder, die einen gemeinsamen Kulturhorizont erinnern, rücken die Bilder des nationalen Mittelalters. Damit tritt die Moderne jener zweideutigen Gestalt näher, in der das verstörte Nebeneinander von nationalkonservativen sowie völkischen Ideologien und zukunftsstürmenden Fortschrittseuphorien sich entfalten wird.

53. Schlegel a.a.O., 178. Zu Schlegels "ästhetischer Hermeneutik" vgl. Heinz-Dieter Weber: Friedrich Schlegels "Transcendentalpoesie". Untersuchungen zum Funktionswandel der Literaturkritik im 18. Jh., München 1973, $156 \mathrm{f}$.

54. Schlegel a.a.O., 190.

55. Vgl. J.Wohlleben: "Germany 1750-1830", in: Dover [Anm.34], 175ff. Zur Konjunktur der philologisch-historischen Antikeforschung in Deutschland vgl. A.Grafton: "Germany and the West 1830-1900", in: Dover [Anm.34], 225ff.; A.Horstmann: Antike Theoria und moderne Wissenschaft. August Boeckhs Konzeption der Philologie, Philologie und Geschichte der Wissenschaften. Studien und Quellen, 17, Frankfurt/M., Berlin etc. 1992.

56. Wie viele seiner Zeitgenossen weist Schlegel selber auf die kulturrelativistische Geltung des Universalismus hin: "Sollte der jetzige französische Nationalcharakter nicht eigentlich mit dem Kardinal Richelieu anfangen? Seine seltsame und beinah abgeschmackte Universalität erinnert an viele der merkwürdigsten französischen Phänomene nach ihm" (a.a.O., 77). 